\title{
Erratum to: Every Banach space admits a homogenous measure of non-compactness not equivalent to the Hausdorff measure
}

\author{
Ehmet Ablet, Lixin Cheng*, Qingjin Cheng \& Wen Zhang \\ School of Mathematical Sciences, Xiamen University, Xiamen 361005, China \\ Email: 619948515@qq.com,lxcheng@xmu.edu.cn,qjcheng@xmu.edu.cn,wenzhang@xmu.edu.cn
}

Erratum to: Science China Mathematics, January 2019 Vol. 62 No. 1: 147-156, https://doi.org/10.1007/s11425-018-9379-y

Citation: Ablet E, Cheng L X, Cheng Q J, et al. Erratum to: Every Banach space admits a homogenous measure of non-compactness not equivalent to the Hausdorff measure. Sci China Math, 2019, 62: 2053-2056, https://doi.org/10.1007/s11425-019-9570-8

[1, Theorem 4.4] states that every infinite dimensional Banach space admits a homogenous measure of noncompactness not equivalent to the Hausdorff measure. Howevere, there is a gap in the proof. In fact, we found that [1, Lemma 4.3] is not true. In this erratum, we give a corrected proof of [1, Theorem 4.4].

For a Banach space $X$, let $\mathscr{C}(X)$ (resp. $\mathscr{B}(X), \mathscr{K}(X))$ be the collection of all non-empty bounded closed convex (resp. nonempty bounded, nonempty convex compact) sets of $X$ endowed with the Hausdorff metric. If there is no confusion, we simply denote them by $\mathscr{C}, \mathscr{B}$ and $\mathscr{K}$, respectively. We use $\Omega$ to denote the closed unit ball $B_{X^{*}}$ of the dual $X^{*}$, and $C_{b}(\Omega)$, the Banach space of all real-valued bounded norm-continuous functions on $\Omega$ endowed with the sup-norm. Let $J: \mathscr{C}(X) \rightarrow C_{b}(\Omega)$ be defined for $C \in \mathscr{C}(X)$ by

$$
J(C)(\omega)=\sigma_{C}(\omega) \equiv \sup _{c \in C}\langle\omega, c\rangle, \quad \omega \in \Omega .
$$

With the symbols as above, we first recall the main results presented in [2], which will be used in the proof.

Theorem 1. (1) (See [2, Theorem 2.3i)]) Given a Banach space $X$, the collection $\mathscr{C}$ consisting of all nonempty closed bounded convex sets of $X$ endowed with the set addition $A \oplus B=\overline{A+B}$, the usual scalar multiplication of sets $\lambda C=\{\lambda c: c \in C\}$, and the norm $\|\cdot|\||$ defined by $\| C\|\mid\| \sup _{c \in C}\|c\|$ is a complete normed convex cone.

(2) (See $\left[2\right.$, Theorem 2.3ii)]) If we endow with the Hausdorff metric $d_{H}$ on $\mathscr{C}$, i.e.,

$$
d_{H}(A, B)=\max \left\{\sup _{a \in A} \inf _{b \in B}\|a-b\|, \sup _{b \in B} \inf _{a \in A}\|b-a\|\right\}
$$

\footnotetext{
* Corresponding author
} 
for all $A, B \in \mathscr{C}$, order $\mathscr{C}$ by set inclusion, and order $C_{b}(\Omega)$ by the usual order of real-valued functions, then the mapping $J: \mathscr{C} \rightarrow C_{b}(\Omega)$ defined for $C \in \mathscr{C}$ and $\omega \in \Omega$ by

$$
J(C)(\omega)=\sigma_{C}(\omega)=\sup _{c \in C}\langle\omega, c\rangle,
$$

is a positively linear order isometry, where $C_{b}(\Omega)$ denotes the Banach space of all bounded norm continuous functions on $\Omega$ endowed with the sup-norm.

(3) (See [2, Theorem 3.2i)]) Both $E_{\mathscr{C}}=\overline{J \mathscr{C}-J \mathscr{C}}$ and $E_{\mathscr{K}}=\overline{J \mathscr{K}-J \mathscr{K}}$ are sublattices of $C_{b}(\Omega)$ and $E_{\mathscr{K}}$ is a lattice ideal of $E_{\mathscr{C}}$, where $\mathscr{K} \subset \mathscr{C}$ is the subcone of $\mathscr{C}$ consisting of all nonempty compact convex subsets of $X$.

(4) (See [2, Theorem 3.2ii)]) The quotient space $Q E_{\mathscr{C}}=E_{\mathfrak{C}} / E_{\mathscr{K}}$ is an abstract $M$ space; hence, it is order isometric to a sublattice of $C(K)$ for some compact Haudorff space $K$.

(5) (See [2, Theorem 3.2iii)]) The subcone TQJC्C is contained in the positive cone of $C(K)$, where $T: Q E_{\mathscr{C}} \rightarrow C(K)$ is an order isometry.

(6) (See [2, Theorem 5.5]) For every bounded subset $F \subset C(K)^{*}$ of positive functionals satisfying that for each $u \in T Q J \mathscr{C}$ there exists $\varphi \in F$ so that $\langle\varphi, u\rangle>0$, the following formula defines a homogenous measure of noncompactness $\mu$ on $X$ :

$$
\mu(B)=\|T[Q J \overline{\mathrm{co}}(B)]\|_{F} \quad \text { for all } B \in \mathscr{B},
$$

where $\|u\|_{F}=\sup _{\varphi \in F \cup-F}\langle\varphi, u\rangle$ for all $u \in C(K)$.

(7) (See [2, Theorem 5.4]) For every bounded subset $F \subset C(K)^{*}$ of

$$
\bigcup_{k \in K}\left\{\mathbb{R}^{+} \delta_{k}: k \in K\right\}=\left\{r \delta_{k}: r \geqslant 0, k \in K\right\}
$$

satisfying that for each $u \in T Q J \mathscr{C}$ there exists $\varphi \in F$ so that $\langle\varphi, u\rangle>0$, the following formula defines a regular measure of noncompactness $\mu$ on $X$ :

$$
\mu(B)=\|T[Q J \overline{\mathrm{co}}(B)]\|_{F} \quad \text { for all } B \in \mathscr{B},
$$

where $\delta_{k}$ is the evaluation functional at $k \in K$,

$$
\|u\|_{F}=\sup _{\varphi \in F \cup-F}\langle\varphi, u\rangle
$$

for all $u \in C(K)$.

(8) (See [2, Theorem 5.4]) In particular, the Hausdorff measure of non-compactness $\beta$ can be reformulated as follows:

$$
\beta(B)=\|T Q J[\overline{\mathrm{co}} B]\|_{C(K)} \text { for all } B \in \mathscr{B} .
$$

Now, we restate and prove the main theorem of [2].

Theorem 2. Every Banach space of infinite dimension admits a homogenous measure of noncompactness not equivalent to the Hausdorff measure.

Proof. Let $X$ be an infinite dimensional Banach space. All symbols will be the same as in Theorem 1 .

If it is a Schur space, i.e., the norm sequential convergence coincides with the weakly sequential convergence on $X$, then we finish the proof by [1, Lemma 3.6].

Suppose that $X$ is not a Schur space. Then it contains a normalized weakly null sequence $\left(y_{n}\right)$. Thus, there is a basic subsequence $\left(x_{n}\right)\left(\subset\left(y_{n}\right)\right)$ of $X$. Without loss of generality, we can assume that $\Delta \equiv\left(x_{n}\right)$ is a monotone basic sequence. (Otherwise, we can renorm $X$ so that $\Delta$ is a monotone basic sequence.) Let $X_{0}=\overline{\operatorname{span}}(\Delta)$. We denote the sequence of the coefficient functionals corresponding to $\Delta$ by $\Delta^{*} \equiv\left(\varphi_{n}\right) \subset X_{0}^{*}$. Then $\left\|\varphi_{n}\right\| \leqslant 2$ and $\varphi_{n} \rightarrow 0$ in the $w^{*}$-topology of $X_{0}^{*} .\left(\Delta_{n}\right)_{n=1}^{\infty}$ be an infintie $\sigma$-partition of $\Delta$, i.e., it satisfies the following conditions: (1) $\bigcup_{n=1}^{\infty} \Delta_{n}=\Delta$; (2) $\Delta_{n} \cap \Delta_{m}=\emptyset$ whenever $n \neq m$; and (3) each $\Delta_{n}$ is an infinite subset of $\Delta$. Let $\left(\Delta_{n}^{*}\right)$ be the partition of $\Delta^{*}$ corresponding to $\left(\Delta_{n}\right)$. For each $n \in \mathbb{N}$, put $C_{n}=\overline{\mathrm{co}}\left(\Delta_{n}\right)$. Then it is easy to observe that 
(a) for each $j \in \mathbb{N}, C_{j}$ is weakly compact, $C_{m} \cap C_{n}=\{0\}$ whenever $m \neq n$, and

(b) for each selection $z_{n} \in C_{n}(n=1,2, \ldots)$ we have $z_{n} \rightarrow 0$ in the weak topology of $X$.

We denote by $C(K)^{+}$(resp. $\left.C(K)^{*+}\right)$ the positive cone of $C(K)\left(\right.$ resp. $\left.C(K)^{*}\right)$. Let

$$
\begin{aligned}
& f_{n}=T Q J\left(C_{n}\right)(\in C(K)), \\
& D=\overline{\operatorname{co}\left(B_{C(K)} \cap C(K)^{+} \cup\left\{n f_{n}: n \in \mathbb{N}\right\}\right)},
\end{aligned}
$$

and let

$$
D^{\circ}=\left\{\varphi \in C(K)^{*+}:\langle\varphi, d\rangle \leqslant 1 \text {, for all } d \in D\right\} .
$$

We claim that $D$ and $D^{0}$ have the following properties:

(i) $D$ contains no nontrivial subcones of $V \equiv T Q J(\mathscr{C})$;

(ii) $D^{0}$ positively separates points of $V$, i.e., for every $0 \neq x \in V$, there is $\varphi \in D^{0}$ such that $\langle\varphi, x\rangle>0$.

To show (i), suppose that $g \in V$ with $g=T Q J(B)$ for some closed bounded nonempty convex set $B$ in $X$ satisfies $n g \in D$ for all $n \in \mathbb{N}$. Then by definitions of $f_{m}, D$ and $D^{0}$, there exists a sequence of $\left(g_{n}\right) \subset V$ with

$$
g_{n}=b_{n}+\sum_{j=1}^{m_{n}} \alpha_{n, j} j f_{j} \in \operatorname{co}\left[B_{C(K)} \cap C(K)^{+} \cup\left\{m f_{m}: m \in \mathbb{N}\right\}\right]
$$

such that $\left\|n g-g_{n}\right\| \rightarrow 0$, where $b_{n} \in B_{C(K)} \cap C(K)^{+}, m_{n} \in \mathbb{N}$, and $\alpha_{n, j} \in \mathbb{R}^{+}$with $\sum_{j=1}^{m_{n}} \alpha_{n, j} \leqslant 1$. Thus

$$
V \ni(1 / n) g_{n}=\frac{b_{n}}{n}+\left(\frac{1}{n}\right) \sum_{j=1}^{m_{n}} \alpha_{n, j} j f_{j} \rightarrow g
$$

(as $n \rightarrow \infty$ ). By Theorems 1(2)-1(5), this is equivalent to that for every $\varepsilon>0$, there exist a finite set $F \subset X$ and $N \in \mathbb{N}$ such that for all $n \geqslant N$,

$$
B \subset\left(\frac{1}{n}\right) \sum_{j=1}^{m_{n}} \alpha_{n, j} j C_{j}+\varepsilon B_{X}+F \quad \text { and } \quad\left(\frac{1}{n}\right) \sum_{j=1}^{m_{n}} \alpha_{n, j} j C_{j} \subset B+\varepsilon B_{X}+F .
$$

Since $(1 / n) \alpha_{n, j} j \rightarrow 0$ for $j=1,2, \ldots$ as $n \rightarrow \infty$, by (b) for every selection

$$
w_{n} \in\left(\frac{1}{n}\right) \sum_{j=1}^{m_{n}} \alpha_{n, j} j C_{j}, \quad n=1,2, \ldots,
$$

we have $w_{n} \rightarrow 0$ in the weak topology of $X$. Consequently, $B \subset \varepsilon B_{X}+F$. Since $\varepsilon$ is arbitrary, $B$ is compact. Consequently, $g=0$.

To show (ii), since $D$ does not contain nontrivial cone of $V$,

$$
D_{1} \equiv \overline{\operatorname{co}\left(D \cup-C(K)^{+}\right)}
$$

does not contain nontrivial subcone of $V$. Assume $f \neq 0 \in V$. Then by (i) we have just provn, $m f \notin V$ for some $m \in \mathbb{N}$. By the separation theorem of convex sets, there is a functional $\varphi \in C(K)^{*}$ such that

$$
\infty>M \equiv\langle\varphi, m f\rangle>\sup \left\{\langle\varphi, d\rangle: d \in D_{1}\right\} \geqslant 0 .
$$

This entails that $\varphi \in C(K)^{*+}$ and bounded above by $M$ on $D$. Let $\psi=\frac{1}{M} \varphi$. Then $\psi \in D^{\circ}$ with $\langle\psi, f\rangle>0$. Thus, (i) and (ii) have been shown. Therefore, by Theorem 1(6),

$$
\mu(B)=\sup _{e \in D^{\circ} \cup-D^{\circ}}\left\langle e, f_{B}\right\rangle, \quad f_{B}=T Q J(\overline{\operatorname{co}(B)}), \quad B \in \mathscr{B}(X)
$$

define a homogenous measure of noncompactness on $X$. Since $\mu\left(C_{n}\right) \leqslant \frac{1}{n} \rightarrow 0$, and since $\beta\left(C_{n}\right) \geqslant \frac{1}{2}$ for all $n \in \mathbb{N}, \mu$ is not equivalent to the Hausdorff measure $\beta$.

Acknowledgements This work was supported by National Natural Science Foundation of China (Grant No. 11731010). 


\section{References}

1 Ablet E, Cheng L, Cheng Q, et al. Every Banach space admits a homogenous measure of non-compactness not equivalent to the Hausdorff measure. Sci China Math, 2019, 62: 147-156

2 Cheng L X, Cheng Q J, Shen Q R, et al. A new approach to measures of noncompactness of Banach spaces. Studia Math, 2016, 240: 21-45

The online version of the original article can be found at https://doi.org/10.1007/s11425-018-9379-y 